\title{
Genetic Variation and Covariation of Yield and Phytochemical Traits in a Red Raspberry Factorial Study
}

\author{
M. Joseph Stephens ${ }^{1}$ \\ The New Zealand Institute for Plant and Food Research Limited, Motueka Research Centre, \\ 55 Old Mill Road, Motueka 7198, New Zealand \\ Jessica Scalzo \\ The New Zealand Institute for Plant and Food Research Limited, Hawke's Bay Research Centre, \\ Cnr Crosses and St. Georges Roads, Private Bag 1401, Havelock North 4157, New Zealand
}
Peter A. Alspach, Ron A. Beatson, and Ann Marie Connor
The New Zealand Institute for Plant and Food Research Limited, Motueka Research Centre, 55 Old Mill Road, Motueka 7198, New Zealand

\begin{abstract}
AdDitIONAL INDEX wORDs. Rubus idaeus, Rubus parvifolius, breeding, heritability, genotypic correlation
ABstract. Breeding for high yield is a major objective of most small fruit breeding programs worldwide. In recent years, research associated with health benefits of berry fruit has resulted in some breeding programs looking for material with improved health properties with the goal of incorporating these into genotypes with high yield and other favorable agronomic characters. In this study, we estimated variance components, heritabilities, and phenotypic and genotypic correlations for yield components and phytochemicals [total phenolics (TPH), antioxidant activity (FRAP), and total anthocyanins (TACY)] from 828 genotypes in a red raspberry (Rubus idaeus) factorial mating design based on 42 full-sib families derived from seven female and six male parents harvested in one season in New Zealand. Narrow sense heritability estimates varied from moderately low [0.23 for percent budburst (PCBB)] to high [0.82 for mean berry weight (BWT)]. Highest genotypic correlations with total yield (TYLD) were found for mean cane length (0.60) followed by moderate correlations for PCBB (0.38), BWT (0.34) and fruit number per unit lateral (0.31). For these four components, the correlation between the product of the empirical breeding values (eBV) and TYLD was as good ( 0.67$)$ as the correlation between the product of the seven yield components measured (0.68). Moderately high negative genotypic correlations were found between TYLD and TPH $(-0.67)$, FRAP $(-0.68)$, and TACY $(-0.64)$, suggesting that breeding for high-yielding genotypes may result in reduced phytochemical levels. A pigment-deficient $R$. parvifolius $\times R$. idaeus hybrid derivative parent ( $R$. parv deriv) had the most influence on berry weight as a yield component. Removal of the $R$. parv deriv progeny from the analysis increased the contribution of berry weight to total yield. Heritability estimates were generally lower when the $\boldsymbol{R}$. parv deriv crosses were omitted, particularly for number of canes BWT, TYLD, and the phytochemicals. Implications of $R$. parv deriv crosses on these results are discussed. Of the 828 genotypes, only five had an $\mathrm{eBV}$ for total yield greater than that of the best yielding parent, whereas 49 had larger berries than the best parent. None of the 828 genotypes had an eBV for yield components or phytochemicals less than that of the lowest parent, whereas $13 \mathrm{had}$ a higher eBV associated with phytochemicals than that of the highest parent.
\end{abstract}

Small fruit breeding programs consider fruit yield as one of their most important commercial objectives. This is especially so in programs working to improve cultivars for the processing industry, where it is often the top breeding objective. Way et al. (1983) reviewed breeding for improved yield in fruit crops. They observed that many current high-yielding fruit cultivars originated from chance seedlings and they suggested that breeders could contribute significant gains by the implementation of systematic breeding strategies. With the use modern statistical tools involving advanced computational procedures applied to quantitative genetic studies in plant breeding programs, there is now real potential to improve fruit crop yield.

Yield is often considered the ultimate quantitative trait (Way et al., 1983). It is estimated to involve many genes thought to

Received for publication 7 Apr. 2009. Accepted for publication 7 Aug. 2009. We thank Harvey Hall who made the crosses for this study and Michael Allred who helped to coordinate the harvest.

${ }^{1}$ Corresponding author. E-mail: jo.stephens@plantandfood.co.nz involve epistatic or pleiotropic effects and hence breeding for yield can be strongly influenced when selecting for other traits. Breeding for high yield involves the interaction of many factors, including genetic, cultural, and environmental as well as the influence of pests and diseases (Daubeny, 1996). Because of this, yield has not been an easy character to improve by conventional breeding procedures. A further difficulty facing breeders is obtaining accurate assessments of yield from breeding population seedlings and advanced selection small plots because of the time-consuming nature of yield measurement. One alternative favored in some red raspberry breeding programs involves yield estimates (Daubeny et al., 1986). Fruit yield can be broken down to several component parts, and accuracy of the yield estimates may be influenced by which components are taken into consideration.

Raspberry plant yield can be increased by optimizing several key components of yield. These include: 1) cane number per plant; 2) fruiting lateral number per cane; 3 ) fruit number per lateral; and 4) fruit size. These four are relatively easily 
measured, but each is determined by contributing traits. Thus, final cane number per plant is influenced by the total number of canes a given plant produces initially and also by the ability of the plant to grow new canes after the application of the springearly summer sucker removal practices, which are commonly used in raspberry production (Waister et al., 1980). Likewise, fruit lateral number per plant is determined by node number, which in turn can be influenced by the cane height and cane diameter (Crandall et al., 1974; Jennings and Dale, 1982) as well as the amount of successful budbreak. Fruit number per lateral is influenced by lateral length and nodes per lateral (Dale, 1976). Fruit size is influenced by ovule number, drupelet size, and number (Dale, 1989). Dale (1989) summarized first-year cane and second-year fruiting yield components for raspberry.

Although all yield components interact with environmental influences to produce total yield, from a breeding point of view, there are certain components that are likely to be better correlated with yield and are more heritable. Dale and Daubeny (1985) showed that high yield in raspberries was closely related to high lateral numbers in Abbotsford, British Columbia, Canada, and thick canes in Invergowrie, Scotland. Several studies have shown that raspberry yield is highly positively correlated with fruit size (Cormack and Woodward, 1977; Dale, 1976; Dale and Daubeny, 1985) and this is probably the easiest component for which breeders can select. Based on circumstantial evidence from raspberry breeding programs around the world, where there has been an increase in average fruit size of released cultivars, it is suggested that this has indeed been achieved (Kempler et al., 2005; Moore, 2004, 2006). However, other yield components may be as important and may hold the key to major advances in fruit yield in the future. Dale (1976) produced a phenotypic correlation matrix for 13 yield components from one raspberry family. He recommended increasing fruit number to improve yield by 1) developing types with increased plant vigor and large numbers of fruit per lateral; and 2) developing plants with average vigor and high fruit numbers per cane.

For numbers of laterals and fruit weight, the quantitative inheritance has been shown to be additive in raspberry (Dale, 1989; Fejer, 1977), which indicates that it could be a character that is relatively easy to improve genetically. Likewise, Daubeny (1996) suggests that other yield components would also be additively inherited in raspberry, but there is limited published work in this area.

Although components key to increasing yield need to be identified and optimized, they cannot be the sole focus of a breeding program. Horticultural characters and consumerdriven traits are also important. The health attributes of berries have become important to consumers fueled by research into the benefits of increased consumption of phenolic and phytochemical compounds in humans. Connor et al. (2005a, 2005b) provide a detailed explanation as to why phenolic compounds were measured in this study. Briefly, Rubus species berries have been found to be good sources of phenolic compounds (Deighton et al., 2000; Wada and Ou, 2002; Wang et al., 1996) and research now is focusing on proving further the health benefits to humans (Ghosh, 2006; Stevenson and Hurst, 2007). One of the key ways in which phenolic compounds contribute health benefits is through antioxidant activity, which can be measured in vitro. As well as phenolic compounds, phytochemicals such as anthocyanins and other flavonols are thought to make up a portion of the antioxidant activity of fruit.
In this study, we measured antioxidant activity, total phenolic content, and total anthocyanins content to determine how breeding for high fruit yield affects health properties of red raspberry and ultimately prospects for the breeder to improve both traits.

An inheritance study, in the form of a factorial mating design, was set up in Motueka, New Zealand, with the aim of: 1) analyzing the effects of several individual yield components on the total fruit yield; 2) determining how the individual components are inherited; 3) ascertaining if there are significant genotypic correlations between various yield components; and 4) estimating the genetic relationship between various yield components and key phytochemicals in the fruit.

\section{Materials and Methods}

Plants. The design, establishment, and maintenance of the factorial mating design are described in detail by Connor et al. (2005b). Briefly, there were 42 families derived from 13 red raspberry cultivars and selections (seven used as female and six as male parents) in The New Zealand Institute for Plant and Food Research Limited (Plant and Food Research, formerly HortResearch) Rubus breeding program. Parents were chosen based on their expected genetic contributions to various fruit and plant characters. All parents exhibited floricane fruiting habit because the breeding programs main objective was to develop floricane types suited to machine harvesting. One female parent, an offspring of an open-pollinated $R$. parvifolius $\times R$. idaeus hybrid, was included for its low chilling requirement, which is a key breeding objective of the New Zealand program. Its fruit was visibly less pigmented than that of other parents. The factorial planting was established at Plant and Food Research's research orchard near Motueka, New Zealand (lat. $41^{\circ} 6^{\prime} \mathrm{S}$, long. $172^{\circ} 58^{\prime} \mathrm{E}$ ) when the plants were 1 year old. Plants were set in a resolvable randomized incomplete Latinized $\alpha$-block design (John and Williams, 1995) with four (fullsib) plants per plot, 13 plots per row (incomplete block), four rows per replication, and four replications of each family (i.e., 16 rows of 52 plants each $=832$ plants in total). Four plants died or did not grow well enough to produce fruit for picking and so were excluded from the analysis. Spacing was $1 \mathrm{~m}$ between plants and $3 \mathrm{~m}$ between rows with plants trained to a three-wire vertical trellis system. All primocanes produced within the main crown of the plant were trained up during winter and tipped at $\approx 15 \mathrm{~cm}$ above the top wire. Standard orchard practices were used for fertilization, weed and pest control, and irrigation.

All yield component data were collected in the 2002-2003 season (hereafter referred to as "2002") when the plants were 3 years old. Before fruit ripening, the lateral length and number of fruit on three randomly chosen laterals from the middle cane region on all plants were measured. Number of fruit per unit length of lateral (NFRT) was calculated. For total plant yield, fruit were harvested 11 times every 4 to $8 \mathrm{~d}$ between 22 Nov. 2002 and 14 Jan. 2003. At each harvest or alternate harvest, 10 berry weights from each plant were determined and mean berry weight calculated.

In June and July 2003, cane number and individual cane length were measured on dormant plants. Three canes per plant were randomly selected and total node number (buds per cane), lateral number (buds that produced a lateral), and length of all laterals were measured. Number of buds per unit length of cane, 
percentage of buds that produced a fruiting lateral (percent budburst), and mean fruiting lateral length were calculated.

The plant data were converted to the following yield components (all lengths are expressed in centimeters): 1) number of canes per plant (NCAN); 2) mean cane length per plant (CLEN); 3) number of buds per centimeter of cane (NBUD); 4) percent budburst (PCBB); 5) mean lateral length (LLEN); 6) NFRT; 7) mean berry weight in grams (BWT); and 8 ) total yield per plant in grams per plant (TYLD).

In the absence of sampling and other errors (e.g., loss of fruit resulting from lateral damage), the product of the first seven components equates to the final one, viz total yield per plant.

For each plant, a loess smooth curve of cumulative yield versus date was interpolated to derive the midharvest date [MHD (date at which $50 \%$ of the final yield had been harvested)] and harvest span [HSP (number of days over which the middle $80 \%$ of the crop was picked)].

Pнyтоснеmicals. Total phenolics (TPH), total anthocyanins (TACY), and ferric-reducing antioxidant power (FRAP) were determined in fruit samples from each of the middle two plants of each four-plant plot. Although they were measured in both the 2002 and 2003 seasons, only data from the 2002 season were used for the current study. TPH content was determined using a Folin-Ciocalteu reagent-based method and results reported as gallic acid equivalents (mg/100 $\mathrm{g}$ fruit). For antioxidant activity, the FRAP (also referred to as ferricreducing ability of plasma) assay was used as modified by Deighton et al. (2000). Results are reported as ferrous sulfate equivalents ( $\mu \mathrm{mol} \cdot \mathrm{g}^{-1}$ fruit). For details on determinations of TPH and total FRAP, see Connor et al. (2005b). Individual anthocyanins were determined by high-performance liquid chromatography and quantified using external standards and Waters Millennium ${ }^{\circledR}$ software (Waters, Milford, MA). TACY were calculated as the sum of individual components and expressed as milligrams per $100 \mathrm{~g}$ fruit. For details on TACY determination, see Connor et al. (2005a).

Statistical APPROACH. Mixed models were fitted to each dependant variate (NCAN, CLEN, NBUD, PCBB, LLEN, NFRT, BWT, TYLD, MHD, HSP, TPH, FRAP, TACY) in turn. The initial model had only random effects: cross, seedling (used here to denote a plant grown from seed), block, subblock within block, and plot within subblock. The numerator relationship matrix was incorporated following the so-called animal model (Lynch and Walsh, 1998), which was considered important given the high level of relationship among the parents. The median coancestry between the parents was 0.08 , the maximum was 0.29 , and over $80 \%$ were nonzero (data not shown). Potential spatial effects were also accommodated in the initial model by fitting a first-order autocorrelation structure (AR1) to both rows and plant position (i.e., 1-52) within row (separate autocorrelation for each). Gilmour et al. (1997) explain this methodology in detail and report that the AR1 structure works well in field trials laid out in a grid structure. This concurs with our experience in various horticultural crops (e.g., Beatson and Alspach, in press). Given the lack of replication at the seedling level, a nugget effect was also included in the model (B. Cullis, personal communication).

Normal probability plots of the residuals were examined to assess whether any data transformation was necessary and which to investigate. Nondesign effects (i.e., cross, seedling, and spatial AR1 effects) in the initial model were retained or dropped based on the magnitudes of the estimates relative to their SES, the likelihood ratio test of nested models, and the row $\times$ plant position semivariograms. For FRAP, the semivariograms suggested the presence of a linear trend along the rows and this was fitted as a fixed effect.

Best linear unbiased predictors were obtained for the seedlings from each univariate analysis; these were the empirical breeding values (eBV) for the seedlings. Because the pedigree information was included, it was also possible to estimate that eBV for each parent. Narrow sense heritabilities were calculated as the ratio between the additive genetic variance and the sum of the residual variance and the additive genetic variance. Genotypic correlations were estimated from the correlations between the eBV. This method is expedient but known to be less than ideal (Lu et al., 2001). Thus, for some pairs of variates, these genetic correlations were compared with those obtained from the additive genetic variance-covariance matrix obtained from fitting bivariate mixed models. In such an analysis, the dependent variate is no longer a column vector but a two-column matrix (one column for each variate) and the incidence matrices and vectors of fixed and random effects are similarly extended (Lynch and Walsh, 1998).

SEs for the heritabilities were estimated following the approximation of Dickerson (1969), which Dieters et al. (1995) found to be justified. For the genotypic correlations, the SES were obtained through a jackknife procedure by successively excluding the eBV of each family in turn when computing the correlation (Buzas, 1997).

The correlation between the product of the eBV for the seven yield component variates and TYLD was calculated. In addition, correlations among TYLD, eBV, and the product of all possible combinations of six-, five-, four-, three-, and twoyield component eBVs were calculated.

All statistical analyses were conducting using R 2.7.0 (R Development Core Team, 2007) and the mixed models were fitted using the ASReml-R package (Butler et al., 2006).

\section{Results}

All final models excluded cross but included seedling as a random effect with the numerator relationship matrix. However, four different final models were used depending on the variate: 1) Model 1 included first-order autocorrelation for both row and plant position and the nugget effect (NCAN, CLEN, NBUD, NFRT, TYLD, HSP, MHD, TPH, and TACY); 2) Model 2 incorporated a linear trend for plant position as fixed effects (FRAP); 3) Model 3 autocorrelation spatial effects excluded (PCBB); and 4) Model 4 autocorrelation row effect excluded [but first-order autocorrelation plant position effect fitted (LLEN and BWT)]. Logarithmic transformations were necessary for both NBUD and NFRT to obtain normally distributed residuals.

The ranges of the parental eBV differed among the various traits: from $\approx 20 \%$ of the mean parental eBV for CLEN, NBUD, and LLEN to over $100 \%$ of the mean parental eBV for TYLD and TACY (Table 1). The eBV of $R$. parv deriv was notably high for CLEN, PCBB, and TYLD and low for BWT, MHD, HSP, and the three phytochemicals (TPH, FRAP, and TACY) (Table 1). Heritability estimates varied from moderately low (0.23 for PCBB) to high (0.91 for FRAP) (Table 2).

Genotypic correlations computed from the correlations between the univariate $\mathrm{eBV}$ and those computed from bivariate analyses were similar (data not shown). The average value of 


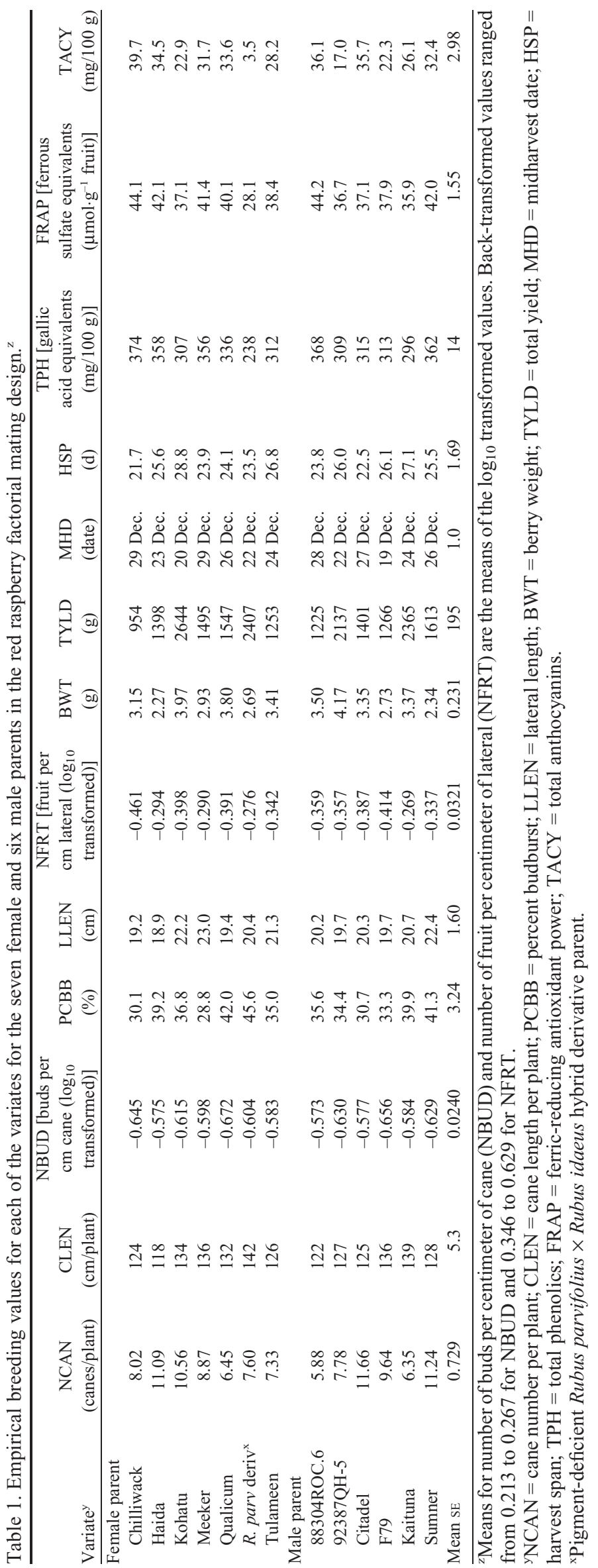

Table 2. Narrow sense heritability estimates and approximate SEs for each variate using genotypes from all crosses ( $n=828$ genotypes) and excluding genotypes from crosses involving the Rubus parvifolius derivative ( $R$. parv deriv) parent $(\mathrm{n}=732$ genotypes) in the red raspberry factorial mating design.

\begin{tabular}{lccccc}
\hline & \multicolumn{2}{c}{ All crosses } & & Excluding R. parv deriv crosses \\
\cline { 2 - 3 } \cline { 5 - 6 } Variate $^{z}$ & Heritability & SE & & Heritability & SE \\
\hline NCAN & 0.64 & 0.280 & & 0.52 & 0.179 \\
CLEN & 0.27 & 0.167 & & 0.32 & 0.178 \\
NBUD & 0.46 & 0.241 & & 0.43 & 0.197 \\
PCBB & 0.23 & 0.116 & & 0.16 & 0.076 \\
LLEN & 0.31 & 0.218 & & 0.27 & 0.187 \\
NFRT & 0.37 & 0.180 & & 0.28 & 0.118 \\
BWT & 0.82 & 0.333 & & 0.58 & 0.145 \\
TYLD & 0.24 & 0.103 & & 0.16 & 0.056 \\
MHD & 0.56 & 0.309 & & 0.41 & 0.161 \\
HSP & 0.77 & 0.367 & & 0.63 & 0.279 \\
TPH & 0.39 & 0.184 & & 0.31 & 0.118 \\
FRAP & 0.41 & 0.185 & & 0.30 & 0.107 \\
TACY & 0.61 & 0.222 & & 0.39 & 0.099
\end{tabular}

${ }^{\mathrm{z}} \mathrm{NCAN}=$ cane number per plant; $\mathrm{CLEN}=$ cane length per plant; NBUD = number buds per cane; $\mathrm{PCBB}=$ percent budburst; LLEN = lateral length; NFRT = number fruit per lateral; BWT = berry weight; TYLD $=$ total yield $;$ MHD $=$ midharvest date; HSP $=$ harvest span; $\mathrm{TPH}=$ total phenolics; FRAP $=$ ferric-reducing antioxidant power; $\mathrm{TACY}=$ total anthocyanins.

the absolute difference between the two methods was 0.12 , which was slightly less than the jackknife SE estimates for most of the correlations ( 0.13 to 0.15 , data not shown). SES for the phenotypic correlations were mostly $\approx 0.035$, except for those involving either harvest span or midharvest date, which were slightly higher at $\approx 0.060$ (data not shown).

Genotypic and phenotypic correlations ranged from negligible to highly negative or positive (Table 3). Of particular interest are those pairs of variates for which the genotypic correlation was considerably different from the phenotypic correlation. Most of these involved the phytochemicals. Phenotypic correlations between TPH, TACY, and FRAP and CLEN, LLEN, TYLD, MHD, and HSP were generally of low magnitude. In contrast, the genotypic correlations of these pairs had magnitudes approaching 0.5 or greater (Table 3 ).

The highest correlation between a single yield component eBV and that of TYLD was 0.60 for mean cane length (phenotypic correlation $=0.47)($ Table 3$)$. The correlation between the product of all seven yield component eBV and TYLD was 0.68 (data not shown). This correlation was matched by the best six (excluding NBUD, $r=0.70$ ), the best five (additionally excluding NCAN, $r=0.71$ ), and the best four (additionally excluding LLEN, $r=0.67$ ). Correlation of TYLD with the best product of three yield components was somewhat lower ( $r=0.60$ for product of CLEN or PCBB and NFRT, and BWT) and that for the best product of two was lower still ( $r=$ 0.53 for product of CLEN and LLEN).

Because the $R$. parv deriv, with its low chill requirement and pale fruit color, may have had considerable influence on the genetic parameters, analyses were repeated omitting crosses involving this parent. Heritability estimates were generally lower in all but one case (CLEN) when these crosses were omitted, particularly for NCAN, BWT, TYLD, and phytochemical traits (Table 2). The exclusion had little impact on the phenotypic correlations, but many genotypic correlations 
Table 3. Phenotypic (upper triangle) and genotypic (lower triangle) correlations between all pairs of variates using genotypes from all crosses ( $\mathrm{n}=$ 828 genotypes) in the red raspberry factorial mating design. ${ }^{\mathrm{z}}$

\begin{tabular}{lrrrrrrrrrrrrr}
\hline Variate $^{\mathrm{y}}$ & NCAN & CLEN & NBUD & PCBB & LLEN & NFRT & BWT & TYLD & MHD & HSP & TPH & FRAP & TACY \\
\hline NCAN & & 0.23 & -0.28 & -0.05 & 0.20 & -0.30 & -0.10 & 0.33 & -0.15 & 0.19 & 0.07 & 0.04 & -0.03 \\
CLEN & -0.10 & & -0.45 & 0.23 & 0.41 & $-\mathbf{0 . 3 1}$ & 0.04 & 0.47 & 0.09 & 0.22 & $\mathbf{0 . 1 1}$ & $\mathbf{0 . 1 0}$ & $-\mathbf{0 . 0 5}$ \\
NBUD & -0.05 & -0.27 & & -0.23 & $-\mathbf{0 . 3 3}$ & 0.32 & -0.05 & -0.22 & -0.08 & -0.14 & 0.01 & 0.01 & 0.15 \\
PCBB & -0.24 & 0.27 & -0.11 & & 0.13 & $-\mathbf{0 . 0 8}$ & -0.04 & 0.30 & -0.01 & 0.05 & 0.01 & 0.02 & -0.02 \\
LLEN & 0.28 & 0.31 & $\mathbf{0 . 1 9}$ & -0.09 & & $-\mathbf{0 . 3 0}$ & 0.10 & 0.41 & 0.17 & 0.13 & 0.01 & -0.01 & -0.11 \\
NFRT & -0.17 & $\mathbf{0 . 2 5}$ & 0.49 & $\mathbf{0 . 3 8}$ & $\mathbf{0 . 2 2}$ & & -0.22 & $-\mathbf{0 . 1 6}$ & -0.03 & -0.09 & 0.02 & 0.04 & 0.06 \\
BWT & -0.30 & 0.01 & -0.12 & -0.12 & -0.05 & -0.28 & & 0.27 & 0.04 & 0.08 & -0.42 & -0.42 & -0.19 \\
TYLD & -0.01 & 0.60 & 0.00 & 0.38 & 0.28 & $\mathbf{0 . 3 1}$ & 0.34 & & -0.18 & 0.28 & $-\mathbf{0 . 0 8}$ & $-\mathbf{0 . 1 0}$ & -0.17 \\
MHD & -0.23 & -0.29 & 0.15 & -0.17 & 0.06 & -0.02 & -0.06 & -0.46 & & -0.62 & $\mathbf{0 . 0 4}$ & $\mathbf{0 . 0 0}$ & $\mathbf{- 0 . 1 4}$ \\
HSP & 0.06 & 0.24 & 0.00 & 0.14 & 0.31 & 0.14 & 0.22 & 0.50 & -0.61 & -0.17 & -0.10 & -0.09 & -0.03 \\
TPH & 0.11 & $-\mathbf{0 . 6 1}$ & -0.06 & -0.30 & 0.02 & -0.33 & -0.17 & $-\mathbf{0 . 6 7}$ & $\mathbf{0 . 5 1}$ & -0.25 & & 0.94 & 0.43 \\
FRAP & 0.03 & $\mathbf{- 0 . 6 2}$ & -0.04 & -0.31 & 0.00 & -0.35 & -0.11 & $-\mathbf{0 . 6 8}$ & $\mathbf{0 . 4 8}$ & -0.18 & 0.98 & \\
TACY & 0.09 & $\mathbf{- 0 . 5 6}$ & 0.07 & -0.35 & -0.02 & -0.33 & -0.04 & -0.64 & $\mathbf{0 . 5 0}$ & -0.18 & 0.83 & 0.85 & 0.47
\end{tabular}

${ }^{\mathrm{z}}$ Bold type marks those in which the difference between the two types of correlation greater than 0.42 (i.e., Bonferroni experimentwise $\alpha$ of 0.05 ). ${ }^{y} \mathrm{NCAN}=$ cane number per plant; $\mathrm{CLEN}=$ cane length per plant; NBUD = number buds per cane; $\mathrm{PCBB}=$ percent budburst; $\mathrm{LLEN}=$ lateral length; NFRT = number fruit per lateral; BWT = berry weight; TYLD = total yield; $\mathrm{MHD}=$ mid harvest date; HSP = harvest span; TPH = total phenolics; FRAP $=$ ferric-reducing antioxidant power; TACY $=$ total anthocyanins.

involving phytochemical traits changed markedly (Fig. 1; Tables 3 and 4). Interestingly, exclusion of these crosses did not reduce the relatively high negative correlations between phytochemicals and TYLD.

Correlations between products of yield component eBV and TYLD eBV were slightly higher when $R$. parv deriv crosses were excluded, most notably for products involving BWT. When these crosses were excluded, all 65 correlations involving BWT were 0.45 or greater and only three correlations 0.45 or greater did not involve BWT (data not shown). In contrast, when all crosses were included, only 50 of the correlations involving BWT were 0.45 or greater and 14 correlations 0.45 or greater did not involve BWT. Thus, the importance of berry weight in determining yield was not as marked when all crosses were included.

Of the 828 seedlings, only five had a higher total yield eBV than the best parent, whereas 49 had larger berries as estimated by the eBV (Table 5). Notably, no seedling had a lower phytochemical eBV than the lowest parent, although up to 13 had a higher phytochemical eBV than the highest parent.

\section{Discussion}

Developing new cultivars that are high yielding is a major goal of raspberry breeding programs around the world. Breeders using recurrent selection practices rely on sufficient yield component heritable variation among and within their seedling populations. There is some evidence suggesting components contributing to total yield are heritable in raspberry (Dale, 1989; Daubeny, 1996; Fejer, 1977), although these authors did not report heritability estimates.

In the absence of sampling and other errors (e.g., loss of fruit resulting from lateral damage), the product of the seven yield components equates to the total yield per plant. Given that these errors are present, the correlation between the product of all components and total yield is likely to be considerably less than one. For example, Kowalenko (2003) found correlations between yield and various yield indices ranging from 0.6 to 0.8 . Our results are consistent with this.

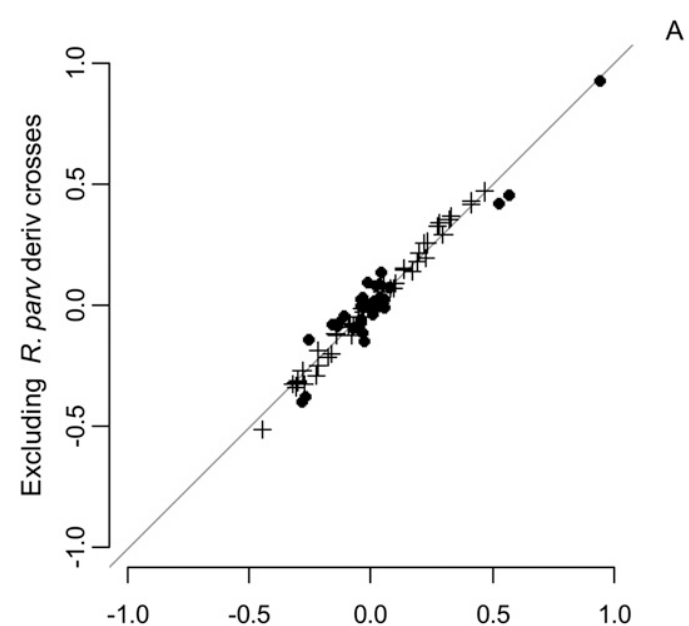

A

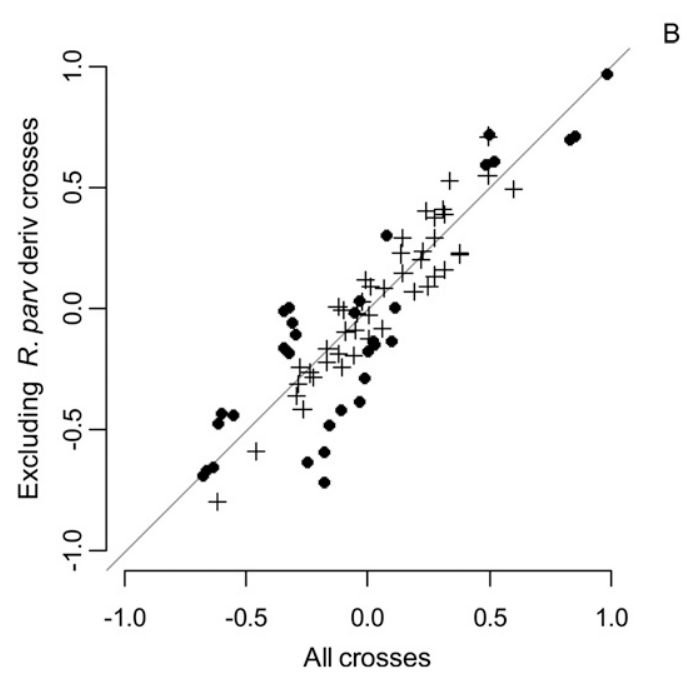

Fig. 1. Scatterplots of correlations excluding Rubus parvifolius derivative ( $R$. parv deriv) crosses on those including all crosses ( $\mathrm{n}=828$ genotypes) in the red raspberry factorial mating design: (A) phenotypic correlations and (B) genotypic correlations. Correlations involving phytochemicals are indicated by filled circles; solid gray line $x=y$. 
Table 4. Phenotypic (upper triangle) and genotypic (lower triangle) correlations between all pairs of variates excluding genotypes from crosses involving the Rubus parvifolius derivative ( $R$. parv deriv) parent $\left(\mathrm{n}=732\right.$ genotypes) in the red raspberry factorial mating design. ${ }^{\mathrm{z}}$

\begin{tabular}{|c|c|c|c|c|c|c|c|c|c|c|c|c|c|}
\hline Variate $^{y}$ & NCAN & CLEN & NBUD & PCBB & LLEN & NFRT & BWT & TYLD & MHD & HSP & TPH & FRAP & TACY \\
\hline NCAN & & 0.26 & -0.27 & -0.04 & 0.22 & -0.32 & -0.09 & 0.37 & -0.12 & 0.18 & 0.08 & 0.04 & -0.03 \\
\hline CLEN & 0.00 & & -0.51 & 0.20 & 0.43 & -0.33 & 0.06 & 0.47 & 0.07 & 0.26 & 0.09 & 0.09 & -0.07 \\
\hline NBUD & -0.02 & -0.41 & & -0.29 & -0.32 & 0.36 & -0.05 & -0.25 & -0.08 & -0.12 & 0.00 & 0.01 & 0.14 \\
\hline LLEN & 0.30 & 0.41 & 0.07 & -0.09 & & -0.31 & 0.09 & 0.42 & 0.14 & 0.15 & 0.02 & 0.02 & -0.09 \\
\hline NFRT & -0.22 & 0.09 & 0.55 & 0.23 & 0.20 & & -0.19 & -0.20 & -0.03 & -0.07 & 0.03 & 0.04 & 0.10 \\
\hline BWT & -0.36 & 0.09 & -0.18 & 0.01 & -0.09 & -0.24 & & 0.33 & 0.01 & 0.07 & -0.40 & -0.37 & -0.14 \\
\hline MHD & -0.28 & -0.31 & 0.15 & -0.16 & -0.08 & 0.03 & -0.19 & -0.59 & & -0.80 & 0.03 & 0.00 & -0.11 \\
\hline HSP & 0.09 & 0.41 & -0.02 & 0.29 & 0.39 & 0.23 & 0.24 & 0.71 & -0.32 & & -0.06 & -0.07 & 0.00 \\
\hline $\mathrm{TPH}$ & 0.01 & -0.43 & -0.01 & -0.10 & -0.13 & -0.18 & -0.48 & -0.67 & 0.61 & -0.63 & & 0.93 & 0.43 \\
\hline FRAP & -0.14 & -0.47 & 0.04 & -0.05 & -0.17 & -0.16 & -0.42 & -0.69 & 0.60 & -0.59 & 0.97 & & 0.46 \\
\hline TACY & -0.13 & -0.44 & 0.31 & 0.00 & -0.28 & 0.01 & -0.38 & -0.65 & 0.73 & -0.71 & 0.70 & 0.72 & \\
\hline
\end{tabular}

${ }^{\mathrm{z} B o l d}$ font marks those in which the difference between the two types of correlation greater than 0.48 (i.e., Bonferroni experimentwise $\alpha$ of 0.05 ). ${ }^{y} \mathrm{NCAN}=$ cane number per plant; CLEN = cane length per plant; NBUD = number buds per cane; PCBB = percent budburst; LLEN = lateral length; NFRT = number fruit per lateral; BWT = berry weight; TYLD = total yield; $\mathrm{MHD}=$ mid harvest date; HSP = harvest span; TPH = total phenolics; FRAP = ferric-reducing antioxidant power; TACY $=$ total anthocyanins.

Table 5. Number of seedlings with variate empirical breeding value $(\mathrm{eBV})>\mathrm{eBV}$ of the highest parent and number with variate $\mathrm{eBV}<$ eBV of the lowest parent using genotypes from all crosses $(n=828$ genotypes) in the red raspberry factorial mating design. ${ }^{\mathrm{z}}$

\begin{tabular}{lrrcrr}
\hline Variate $^{\mathrm{y}}$ & Greater & Less & Variate $^{\mathrm{y}}$ & Greater & Less \\
\hline NCAN & 7 & 28 & MHD & 22 & 10 \\
CLEN & 1 & 2 & HSP & 0 & 7 \\
NBUD & 41 & 1 & TPH & 6 & 0 \\
PCBB & 2 & 13 & FRAP & 5 & 0 \\
LLEN & 2 & 4 & TACY & 13 & 0 \\
NFRT & 16 & 2 & & & \\
BWT & 49 & 21 & & & \\
TYLD & 5 & 1 & & &
\end{tabular}

${ }^{\mathrm{z}}$ Values in italic font are unlikely to be of practical interest.

${ }^{\mathrm{y}} \mathrm{NCAN}=$ cane number per plant; CLEN = cane length per plant; NBUD = number buds per cane; $\mathrm{PCBB}=$ percent budburst; LLEN = lateral length; NFRT = number fruit per lateral; BWT = berry weight; TYLD = total yield; MHD = mid harvest date; HSP = harvest span; $\mathrm{TPH}=$ total phenolics; FRAP $=$ ferric-reducing antioxidant power; TACY $=$ total anthocyanins.

Using factorial mating design data from a single year in a single location in New Zealand, we have shown that TYLD has relatively low heritability $\left(h^{2}=0.24\right)$. However, several individual yield components such as NCAN $\left(h^{2}=0.64\right)$ and BWT $\left(h^{2}=0.82\right)$ were highly heritable, suggesting that breeders could make significant fruit yield gains by selecting for these individual components. However, negative genotypic correlations between some of these components could hinder progress. The highest genotypic correlations with TYLD were found among CLEN (0.60), PCBB (0.38), BWT (0.34), and NFRT (0.31). Indeed, these four yield components alone were as good an indicator of TYLD (correlation between the product of four components eBV and TYLD was 0.67 ) as the seven measured in this study (correlation between the product of all components eBV and TYLD was 0.68). These findings then indicate that an effective breeding strategy for increasing yield (at least in this location in New Zealand) would be to prioritize these four traits (CLEN, PCBB, NFRT, and BWT). It needs to be remembered that our study only includes data from one season and so we gained no impression of how big of an effect this environmental component might have on our results. However, the 2002 season was not unusual in any way in terms of temperature and rainfall normally experienced in this location. Our results are consistent with those of Dale and Daubeny (1985) who found that total yield is highly correlated with lateral number per cane (equivalent to PCBB in this study) in a clonal cultivar trial in Abbotsford, British Columbia, Canada. Likewise, high correlation of BWT with TYLD was found by both Dale (1976) and Dale and Daubeny (1985).

Of the four components that contributed most significantly to yield, only BWT was highly heritable $\left(h^{2}=0.83\right)$. The heritabilities of the remaining components were comparatively low $\left(h^{2}=0.23\right.$ to 0.37$)$, suggesting progress will be more difficult to achieve. Nevertheless, given the importance of their contribution to yield, seedling selection based solely on BWT, although easy and carrying some merit, is not advisable, especially because selecting for BWT could have a negative impact on other components such as NCAN and NFRT. CLEN, PCBB (or lateral number), and NFRT should be considered as well. It is likely that a selection index approach, using the combined genetic information for the four components (mentioned previously), will maximize the chances of success. The general formula for deriving a selection index is based on the variance-covariance matrix (Falconer and Mackay, 1996):

$$
\begin{aligned}
& \mathrm{Pb}=\mathrm{Ga}, \\
& \text { which rearranges to } \mathrm{b}=\mathrm{P}^{-1} \mathrm{Ga} \text {, }
\end{aligned}
$$

where $P$ and $G$ are the phenotypic and genetic variancecovariance matrices, respectively, a is the vector of weights chosen by the breeder, and $b$ is the vector of index coefficients to be applied to the phenotypic values.

When $R$. parv deriv crosses were excluded from analysis, the effect of some yield components on TYLD increased. This was most pronounced for BWT. This was probably because $R$. parv deriv crosses had a greater proportion of progeny with high yield and not such large fruit. The $R$. parv deriv parent itself is 
well adapted to low chill environments and has high percent budbreak and hence high lateral numbers. It is suggested that PCBB is likely to have had a greater influence over total yield in these $R$. parv deriv progeny than BWT. Therefore, selection for large-fruited types in such populations might be less effective at increasing yield than in populations not involving the $R$. parv deriv parent.

Using data from a single year, we found moderate heritabilities for TPH, TACY, and FRAP. Excluding $R$. parv deriv crosses from the analysis generally reduced the heritability estimates. Connor et al. (2005a), using 2 years of data from the same factorial mating design, also reported lower heritabilities for individual and total anthocyanins and for antioxidant activity when $R$. parv deriv crosses were excluded.

We found moderately high negative genotypic correlations between phytochemical traits and TYLD despite low phenotypic correlations. We found no reports in the literature on the interaction of fruit phytochemical composition and yield in small fruit. Our results suggest breeding solely for high total phenolic, high antioxidant, or high total anthocyanin could have a negative effect on yield and vice versa. However, genotypic correlations of total phytochemical composition and yield components (including BWT) were generally small except for CLEN (the greatest contributor to total yield), which were large and negative. These results are consistent with Connor et al. (2005b), who also found low genotypic correlations between TPH and FRAP and BWT and concluded that progress could be made in breeding for improved fruit weight and antioxidant properties. This did not apply when $R$. parv deriv progeny are excluded from the analysis, which resulted in negative genotypic correlations between BWT and TPH and FRAP. A breeder might need to be cautious when drawing conclusions from this study when including $R$. parv deriv progeny, because the genetic makeup of this material differs significantly from that of $R$. idaeus of the rest of the trial and could be classed as a "subline," which might have a different breeding strategy applied to it.

Exclusion of $R$. parv deriv progeny did not reduce negative genotypic correlations between TPH, TACY, or FRAP and TYLD. Additionally, the exclusion only partially reduced the negative correlations between phytochemical traits and cane length; and it increased the negative correlation between phytochemical traits and berry weight. Therefore, it is suggested that breeding in this environment (Motueka, New Zealand) for increased yield may result in lower phytochemical content and antioxidant activity. Again, a selection index approach mentioned previously for the yield components may be an effective strategy to improve both yield and phytochemicals in genotypes from seedling populations genetically and simultaneously. Another consideration is that antioxidant phytochemicals such as anthocyanins and other phenolics have a protective role against biotic (e.g., disease) and abiotic (e.g., ultraviolet light) stresses in plants. Photosynthate used for polyphenol production would thus be unavailable for vegetative growth and fruit production. These interactions might contribute to the changes (or lack thereof) in correlation observed with the exclusion of phytochemically deficient crosses.

Other genotypic correlations associated with the phytochemicals were altered when $R$. parv deriv crosses were excluded. A moderate genotypic correlation was found between MHD and phytochemical composition and antioxidant capacity when all crosses were included. This could be because some later-ripening genotypes had higher phenolics and antioxidant activity. Parents 'Meeker', 'Tulameen', and 'Citadel' are later ripening and have high anthocyanins and antioxidant activity (Connor et al., 2005a). The genotypic correlations increased when $R$. parv deriv crosses were removed from the analysis, probably because of the low fruit pigment content of these seedlings.

Using the same factorial mating design, Connor et al. (2005b) reported a heritability of $h^{2}=0.72$ for berry weight for the 2002 season. Means were calculated from 100-g samples of fruit. Their result is consistent with the heritability in our study $\left(h^{2}=0.82\right)$ in which mean berry weight was calculated from fruit picked for yield. In their report, Connor et al. (2005b) noted that calculation of heritability did not adjust for common ancestry of parents in the factorial, which was accounted for in our analysis. Connor et al. (2005b) reported a heritability of $h^{2}=0.55$ for TPH using 2002 data, whereas Connor et al. (2005a) reported a heritability of $h^{2}=1.00$ for TACY for combined year (2002 and 2003) data. This contrasts with $h^{2}=39$ and $h^{2}=0.61$ for TPH and TACY, respectively, in this analysis using 2002 data. The differences are probably explained by the use of a different model in our approach, including fitting spatial correlations and the coancestry matrix, which should provide more accurate estimates.

It is recognized that data used in this study were for 2002 only and that Connor et al. (2005a, 2005b) found significant year effects for TPH, FRAP, and TACY using the same factorial. However, they also found the year effect to be less than the genetic effects, and the year $\times$ genotype effects were not significant in their study. Thus, although the breeding values for the phytochemicals might differ between years, the rankings of these breeding values (and hence selection decisions based on them) are likely to be reasonably stable. Whether this also applies to the yield component variates cannot be determined from our data and remains a matter for speculation.

In summary, the study found that total yield and some of the four most important yield components were moderately to highly heritable. Moderately negative genotypic correlations were found between phytochemical traits and total yield suggesting development of high-yielding types high in phytochemicals might be harder to achieve. The use of selection indices is suggested as a method of breeding for higher yield and phytochemical levels in red raspberry.

\section{Literature Cited}

Beatson, R.A. and P.A. Alspach. The use of empirical breeding values to improve genetic progress in hops. Acta Hort. (in press).

Butler, D.G., B.R. Cullis, A.R. Gilmour, and B.J. Gogel. 2006. ASReml-R reference manual, release 2. Queensland Department of Primary Industries and Fisheries, Brisbane, Australia.

Buzas, J.S. 1997. Fast estimators of the jackknife. Am. Stat. 51:235240.

Connor, A.M., T.K. McGhie, M.J. Stephens, H.K. Hall, and P.A. Alspach. 2005a. Variation and heritability estimates of anthocyanins and their relationship to antioxidant activity in a red raspberry factorial mating design. J. Amer. Soc. Hort. Sci. 130:534-542.

Connor, A.M., M.J. Stephens, H.K. Hall, and P.A. Alspach. 2005b. Variation and heritabilities of antioxidant activity and total phenolic content estimated from a red raspberry factorial experiment. J. Amer. Soc. Hort. Sci. 130:403-411.

Cormack, M.R. and P.J. Woodward. 1977. Raspberry cultivar assessments at the national fruit trials and the Scottish Horticultural Research Institute. Expt. Hort. 29:1-14. 
Crandall, P.C., J.D. Chamberlain, and K.A. Biderbost. 1974. Cane characteristics associated with berry number of red raspberry. J. Amer. Soc. Hort. Sci. 99:370-372.

Dale, A. 1976. Prospects for breeding higher yielding raspberries. Acta Hort. 60:159-167.

Dale, A. 1989. Productivity in red raspberries. Hort. Rev. (Amer. Soc. Hort. Sci.) 11:185-227.

Dale, A. and H.A. Daubeny. 1985. Genotype-environment interactions involving british and pacific northwest red raspberry cultivars. HortScience 20:68-69.

Daubeny, H.A. 1996. Brambles, p. 109-190. In: J. Janick and J.N. Moore (eds.). Fruit breeding, vine and small fruits. Wiley, New York, NY.

Daubeny, H.A., A. Dale, and G. McGregor. 1986. Estimating yields of red raspberries in small research plots. HortScience 21:1216-1217.

Deighton, N., R. Brennan, C.E. Finn, and H.V. Davies. 2000. Antioxidant properties of domesticated and wild Rubus species. J. Sci. Food Agr. 80:1307-1313.

Dickerson, G.E. 1969. Techniques for research in quantitative animal genetics, p. 36-79. In: A.B. Chapman (ed.). Techniques and procedures in animal science research. American Society of Animal Science, Albany, NY.

Dieters, M.J., T.L. White, R.C. Littell, and G.R. Hodge. 1995. Application of approximate variances of variance components and their ratios in genetic tests. Theor. Appl. Genet. 91:15-24.

Falconer, D.S. and T.F.C. Mackay. 1996. Introduction to quantitative genetics. Longmans Green, Harlow, UK.

Fejer, S.O. 1977. Inheritance of yield, yield components, and fall-fruiting habit in red raspberry diallel crosses. Can. J. Genet. Cytol. 19:1-13.

Ghosh, D., T.K. McGhie, J. Zhang, A. Adaim, and M. Skinner. 2006. Effects of anthocyanins and other phenolics of boysenberry and blackcurrant as inhibitors of oxidative stress and damage to cellular DNA in SH-SY5Y and HL-60 cells. J. Sci. Food Agr. 86:678-686.

Gilmour, A.R., B.R. Cullis, and A.P. Verbyla. 1997. Accounting for natural extraneous variation in analysis of field experiments. J. Agr. Biol. Ecol. Stat. 2:269-293.
Jennings, D.L. and A. Dale. 1982. Variation in the growth habit of red raspberries with particular reference to cane height and node production. J. Hort. Sci. 57:197-204.

John, J.A. and E.R. Williams. 1995. Cyclic and computer generated designs. Chapman and Hall, London, UK.

Kempler, C., H.A. Daubeny, B. Harding, and C.G. Kowalenko. 2005. 'Cowichan' red raspberry. HortScience 40:1916-1918.

Kowalenko, C.G. 2003. An evaluation of estimating and indexing methods to simplify the determination of management treatment effects on raspberry yields. Can. J. Plant Sci. 83:141-147.

Lu, P., D.A. Huber, and T.L. White. 2001. Comparison of multivariate and univariate methods for estimation of type B genetic correlations. Silvae Genet. 50:13-22.

Lynch, M. and B. Walsh. 1998. Estimation of breeding values, p. 745778. In: M. Lynch and B. Walsh (eds.). Genetics and analysis of quantitative traits. Sinauer Associates, Sunderland, MA.

Moore, P.P. 2004. 'Cascade Delight' red raspberry. HortScience 39:185-187.

Moore, P.P. 2006. 'Cascade Dawn' red raspberry. HortScience 41: 857-859.

R Development Core Team. 2007. R: A language and environment for statistical computing. 5 Aug. 2009. <http://www.R-project.org>.

Stevenson, D.E. and R.D. Hurst. 2007. Polyphenolic phytochemicalsJust antioxidants or much more? Cell. Mol. Life Sci. 64:29002916.

Wada, L. and B. Ou. 2002. Antioxidant activity and phenolic content of Oregon caneberries. J. Agr. Food Chem. 50:3495-3500.

Waister, P.D., C.J. Wright, and M.R. Cormack. 1980. Potential yield in red raspberry as influenced by interaction between genotype and cultural methods. Acta Hort. 112:273-283.

Wang, H., G. Cao, and R.L. Prior. 1996. Total antioxidant capacity of fruits. J. Agr. Food Chem. 44:701-705.

Way, R.D., J.C. Sanford, and A.N. Lasko. 1983. Fruitfulness and productivity, p. 353-367. In: J.N. Moore and J. Janick (eds.). Methods in fruit breeding. Purdue University Press, West Lafayette, IN. 